\title{
Avaliação radiográfica da consolidação das fraturas desviadas do colo cirúrgico do úmero tratadas com fixação percutânea*
}

\section{Radiographic Evaluation of the Consolidation of Humerus Surgical Neck Fractures Treated With Percutaneous Fixation}

\author{
Ivan Fadanelli Simionato ${ }^{1}$ João Pedro Farina Brunelli ${ }^{1}$ Marco Tonding Ferreira ${ }^{1}$ \\ Fabio Yoshihiro Matsumoto ${ }^{1}$ Almiro Gerszon Britto ${ }^{1}$ Fernando Carlos Mothes ${ }^{1}$
${ }^{1}$ Grupo de Cirurgia do Ombro e Cotovelo, Santa Casa de Misericórdia de Porto Alegre, RS, Brasil
Endereço para correspondência Ivan Fadanelli Simionato, MD, Rua Barão do Cotegipe 124/202, Bairro São João, Porto Alegre, 90540020 , Rev Bras Ortop 2020;55(3):347-352. Brasil (e-mail: Simionato.md@gmail.com).

\section{Resumo \\ Palavras-chave \\ - fraturas do úmero \\ - fraturas do colo umeral/cirurgia \\ - fixação interna de fraturas \\ - radiografia}

Objetivo Avaliar radiograficamente os resultados pós-operatórios dos pacientes com fratura da extremidade proximal do úmero em duas partes do colo cirúrgico tratados com fixação percutânea com fios rosqueados.

Métodos Avaliamos radiograficamente os resultados da fixação percutânea com fios rosqueados das fraturas da extremidade proximal do úmero realizadas em nosso serviço. Foram avaliadas as imagens pré- e pós-operatórias de 42 pacientes, avaliandose radiografias com até 8 semanas de pós-operatório. Consideramos na avaliação o desvio da fratura pré-operatório, a perda da redução e a consolidação da fratura.

Resultados O índice de consolidação nos casos avaliados foi de $90,4 \%$, com perda da redução em $16,6 \%$ dos casos e não obtida a consolidação em apenas 4 casos. Foi observada uma predominância de determinado padrão de fratura nos casos que perderam a redução.

Conclusão $\mathrm{O}$ estudo permitiu considerar a técnica de fixação percutânea com fios rosqueados uma alternativa no arsenal terapêutico da fratura da extremidade proximal do úmero do colo cirúrgico, com contraindicação para os traços de fratura baixos na metáfise e com acometimento da cortical medial/lateral.

Objective To evaluate radiographically the postoperative results of patients with fracture of the proximal humerus in two parts of the surgical neck treated with threaded percutaneous fixation.

Methods We evaluated the radiographic results of percutaneous fixation with threaded pins on the treatment of proximal humerus fractures in our service. Preoperative and postoperative images of 42 patients were evaluated, evaluating

Trabalho feito no Grupo de Cirurgia do Ombro e Cotovelo, Santa

Casa de Misericórdia de Porto Alegre, RS, Brasil

received

January 13, 2018

accepted

May 2, 2019
DOI https://doi.org/

10.1055/s-0039-3402463. ISSN 0102-3616.
Copyright $\odot 2020$ by Sociedade Brasileira License terms de Ortopedia e Traumatologia. Published by Thieme Revinter Publicações Ltda, Rio de Janeiro, Brazil 
Keywords

- humeral fractures

- humeral neck fractures/surgery

- fracture fixation internal

- radiography radiographs until 8 weeks postoperatively. We considered on evaluation the fracture deviation on preoperative images, the loss of reduction and no consolidation.

Results Our consolidation rate was $90.4 \%$, with loss of reduction in $16.6 \%$ of the cases and no consolidation rates in just 4 operated cases. We observed a predominance of a specific fracture pattern in the cases with loss of reduction.

Conclusion The present study allows us to consider the percutaneous fixation technique with threaded pins as an alternative in our therapeutic arsenal for the proximal humerus fracture of the surgical neck. Contraindications are considered for low fracture trait on the metaphysis and with medial/lateral cortical impairment.

\section{Introdução}

As fraturas do úmero proximal são extremamente comuns na prática ortopédica. É esperado que sua incidência aumente ainda mais devido à longevidade e maior atividade da população idosa. Segundo Horak et $\mathrm{al}^{1}$, representam $5 \%$ de todas as fraturas. ${ }^{1} \mathrm{O}$ tratamento conservador de fraturas deslocadas não apresenta bons resultados, sendo o tratamento cirúrgico o de escolha na maioria dos casos. ${ }^{2,3}$ Apesar de comuns, essas fraturas ainda são consideradas um desafio terapêutico, com uma extensa gama de técnicas de fixação, mas ainda sem evidências claras de superioridade entre algumas delas, especialmente se considerarmos a ampla variabilidade de características próprias de cada paciente e do padrão de fratura. ${ }^{4} \mathrm{O}$ emprego de implantes rígidos, amplamente utilizados, pode não ser adequado para alguns pacientes, especialmente para ossos osteoporóticos. ${ }^{2,4,5}$ As cirurgias artroplásticas não conseguiram obter melhores resultados funcionais em relação às reconstrutivas, sendo resguardadas, em sua maioria, como métodos de salvamento para falhas de síntese. ${ }^{2,6,7} \mathrm{O}$ conceito de compactação controlada, muito bem demonstrado por Resch et $\mathrm{al}^{7}$ em diferentes estudos, reforça esse conceito. E, para alguns autores, 0 implante ideal seria um implante semirrígido que permita o processo de compactação óssea e remodelamento da fratura que ocorre durante o processo de consolidação e ao mesmo tempo oferece estabilidade a osteossíntese. ${ }^{2,8-11}$ Respaldados pela literatura e baseado nesses princípios, consideramos que o emprego de fios rosqueados, respeitando conceitos de impacção controlada/fixação semi-rígida, é uma técnica adequada para tratamento das fraturas do úmero proximal.

O objetivo deste trabalho foi avaliar, primariamente, a consolidação das fraturas do úmero proximal através de exame radiográfico dos pacientes tratados com fixação percutânea com fios rosqueados. E, de maneira secundária, identificar aspectos que predisponham a uma perda da redução.

\section{Material e Métodos}

Foram avaliados retrospectivamente 46 pacientes com fratura aguda da extremidade proximal do úmero, compreendendo até 2 semanas do dia do trauma, submetidos ao tratamento cirúrgico envolvendo a redução e fixação percutânea com fios rosqueados de $3.5 \mathrm{~mm}$. Desse total, foram excluídos quatro pacientes que, embora tenham sido tratados com a técnica em questão, apresentavam fraturas em três partes, sem necessidade de intervenção na fratura do tubérculo maior após a redução fechada, formando nossa amostra com 42 pacientes dentro dos critérios do estudo. Todos os pacientes foram operados pelos quatro cirurgiões seniores do serviço. A amostra foi composta por 35 mulheres e 7 homens com no mínimo 2 meses de pós-operatório, sendo 11 pacientes do sistema público de saúde e 31 pacientes do sistema privado. A redução e o posicionamento dos fios rosqueados foram feitos sob visualização radiológica intraoperatória com intensificador de imagem. Avaliou-se a manutenção da redução e consolidação da fratura através de incidências radiográficas de anteroposterior, perfil de escápula e Velpeau View, a qual é uma alternativa à realização da incidência axilar sem necessidade de retirar o membro da tipoia, realizando-se a incidência com o paciente fazendo uma inclinação de $20^{\circ}$ a $30^{\circ}$ para posterior com o tronco sob o chassi, que fica localizado inferiormente ao ombro e o feixe posicionado superiormente ao ombro perpendicular ao plano horizontal (- Figura 1), realizadas na $1^{\mathrm{a}}, 2^{\mathrm{a}}$ e $4^{\mathrm{a}}$ semanas de pós-operatório. Os fios rosqueados foram retirados entre a $4^{\mathrm{a}}$ e $5^{\mathrm{a}}$ semana após o procedimento. Além disso, foram realizadas radiografias na $2 a$, 4a e $8^{\text {a }}$ semana após a retirada dos pinos (- Figuras 2-5). Os pacientes foram mantidos em imobilização com tipoia tipo Velpeau durante o pós-operatório até a retirada dos fios, iniciando movimentos passivos de ombro somente após a retirada dos implantes. Considerou-se como não consolidação os casos que, após o período de 4 a 5 semanas, necessitaram de reintervenção e os casos que evoluíram para pseudoartrose. A perda de redução foi considerada como qualquer desvio do colo cirúrgico do úmero observado nas radiografias pós-operatórias, ocorrido até a retirada dos fios, comparando-se com a imagem radiográfica realizada no transoperatório.

\section{Resultados}

Dos 42 pacientes selecionados para o estudo, a média de idade foi 58,5 anos, e a mediana foi de 63,5 anos (- Tabela $\mathbf{1}$ ). Do total de 42 pacientes, 38 obtiveram consolidação da fratura $(90,4 \%)$ (-Tabela 2). Do total, 35 pacientes mantiveram a redução $(83,3 \%)$ e 7 pacientes perderam a redução $(16,6 \%)$ ( - Tabela 3). Dentre os 7 pacientes que perderam a redução obtida na sala cirúrgica, apenas 3 não apresentaram 


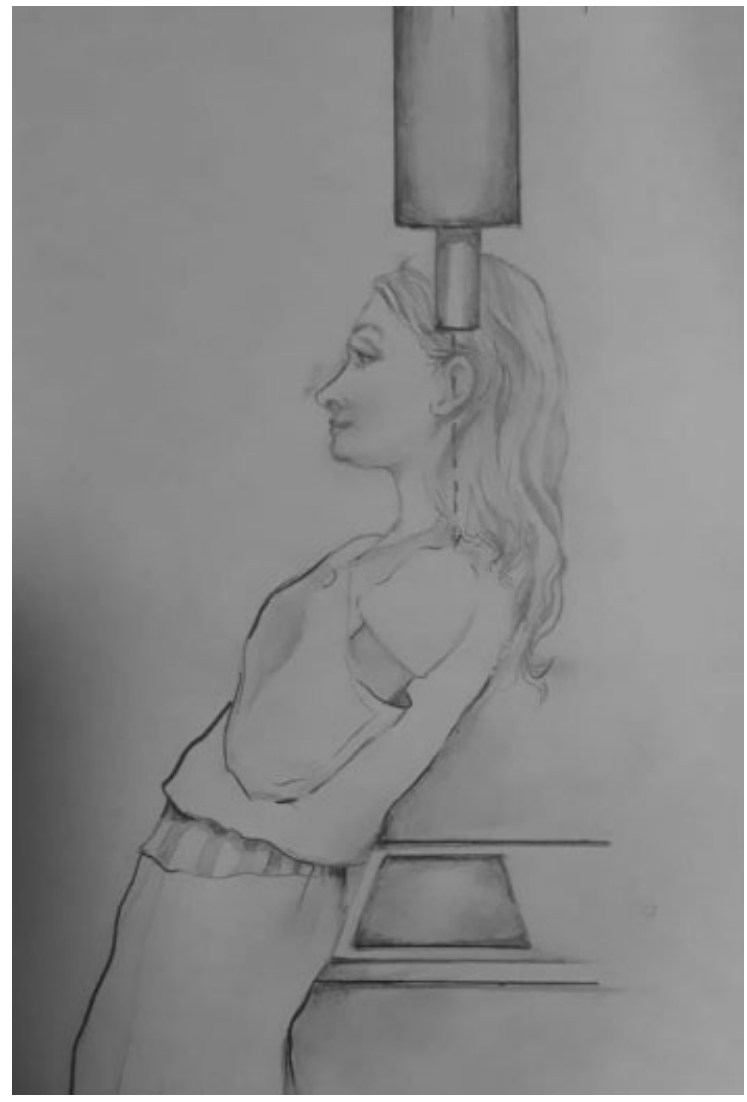

Fig. 1 Incidência radiográfica Velpeau View.

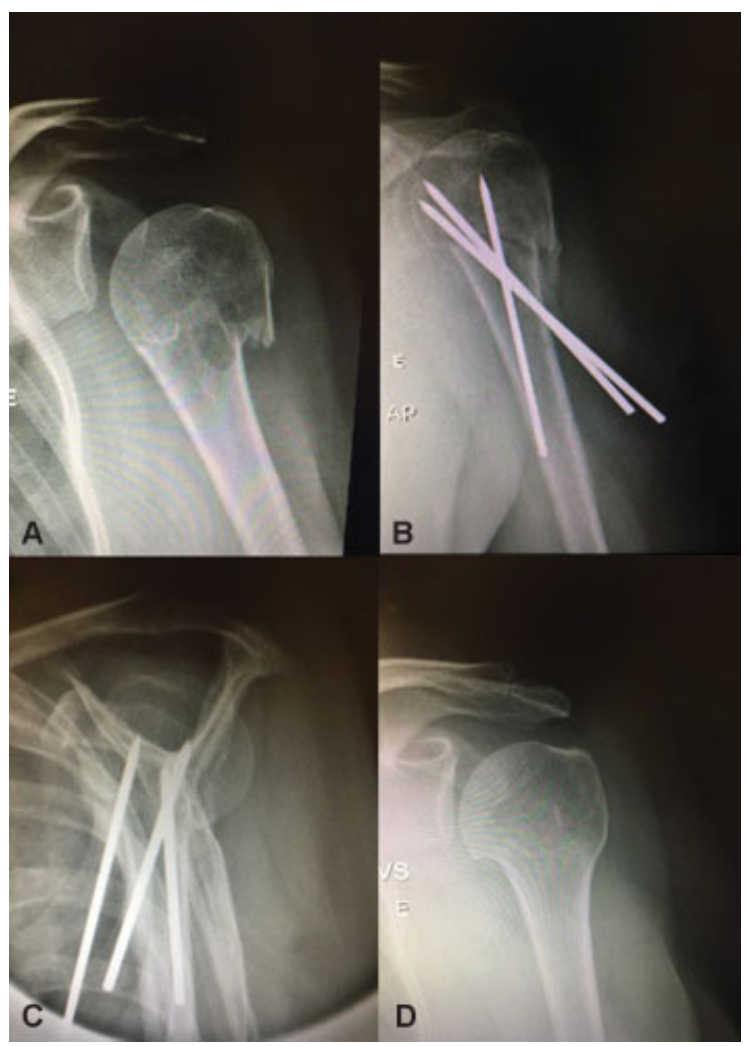

Fig. 2 Radiografia pré-operatória (A); Radiografias pós-operatórias com 4 semanas (B, C); Radiografia pós-operatória com 2 meses (D).
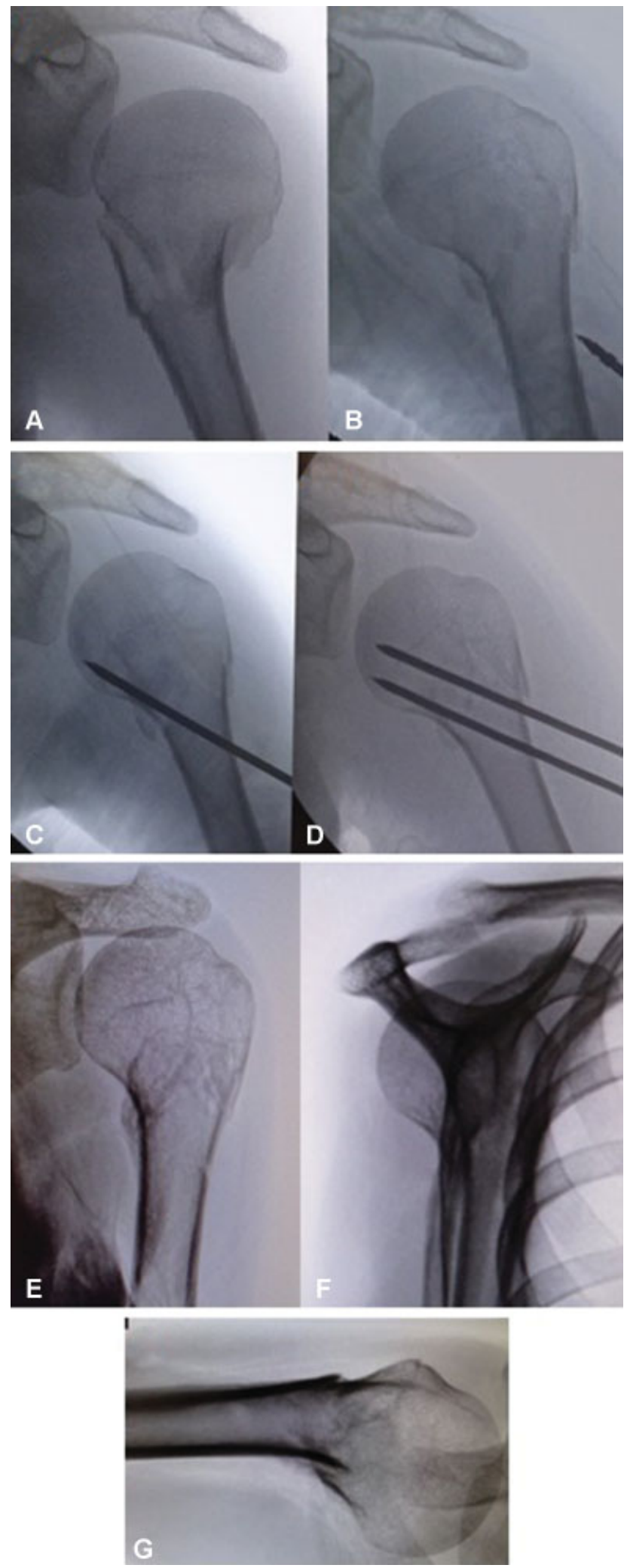

Fig. 3 Radiografia pré-operatória (A); Radiografias transoperatórias (B, C, D); Radiografia pós-operatória com 4 meses (E, F, G).

consolidação da fratura (42,8\%). Em todos os casos de perda da redução, houve a medialização do colo cirúrgico após a fixação com os pinos. Os três pacientes que não consolidaram foram reoperados com placa bloqueada de úmero proximal (-Figura 6). Um paciente apresentou perda da redução no 


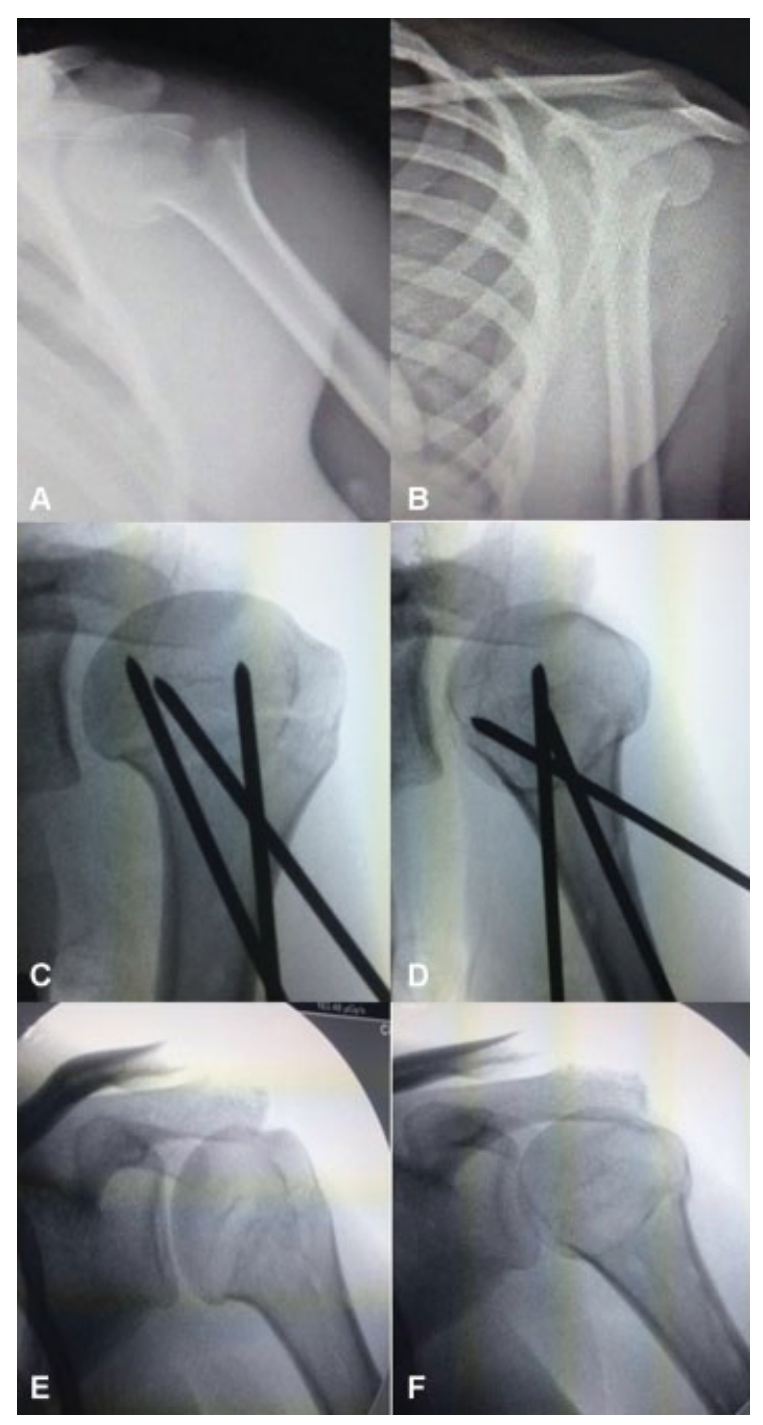

Fig. 4 Radiografia pré-operatória (A, B); Radiografias transoperatórias (C, D); Radiografia pós-operatória com 4 meses (E, F).
Tabela 1 Idade dos pacientes

\begin{tabular}{|l|l|}
\hline Idade media (anos) & 58,47619048 \\
\hline Idade mediana (anos) & 63,5 \\
\hline Intervalo de idade (anos) & $15-85$ \\
\hline
\end{tabular}

Tabela 2 Taxa de consolidação em porcentagem

\begin{tabular}{|l|l|l|}
\hline \multicolumn{2}{|l|}{ CONSOLIDAÇ̃̃O } \\
\hline SIM & NÃO & TOTAL \\
\hline 38 & 4 & 42 \\
\hline 0,904 & 0,095 & 1 \\
\hline
\end{tabular}

Tabela 3 Taxas de perda da redução em porcentagem

\begin{tabular}{|l|l|l|}
\hline \multicolumn{2}{|l|}{ PERDA DA REDUÇ̃̃O } \\
\hline SIM & NÃO & TOTAL \\
\hline 7 & 35 & 42 \\
\hline 0,166667 & 0,83333333 & 1 \\
\hline
\end{tabular}

pós-operatório imediato e foi reintervido em menos de 24 horas com redução aberta e fixação com fios rosqueados, evoluindo para consolidação, sendo, porém, excluído do grupo de bons resultados. Sendo assim, 4 pacientes do estudo foram incluídos no grupo de não consolidação (9,5\%). A média de idade dos pacientes que mantiveram a redução foi de $56,6( \pm 17,6)$ anos, dos que perderam a redução foi de $66,7( \pm 15,7)$ anos e dos que não consolidaram foi de 73,6 $( \pm 8,6)$ anos ( - Tabela 4). Os casos nos quais houve a perda da redução apresentavam traço mais distal da fratura, alguns com cominuição dos fragmentos, em relação aos casos nos quais se manteve a redução no pós-operatório.

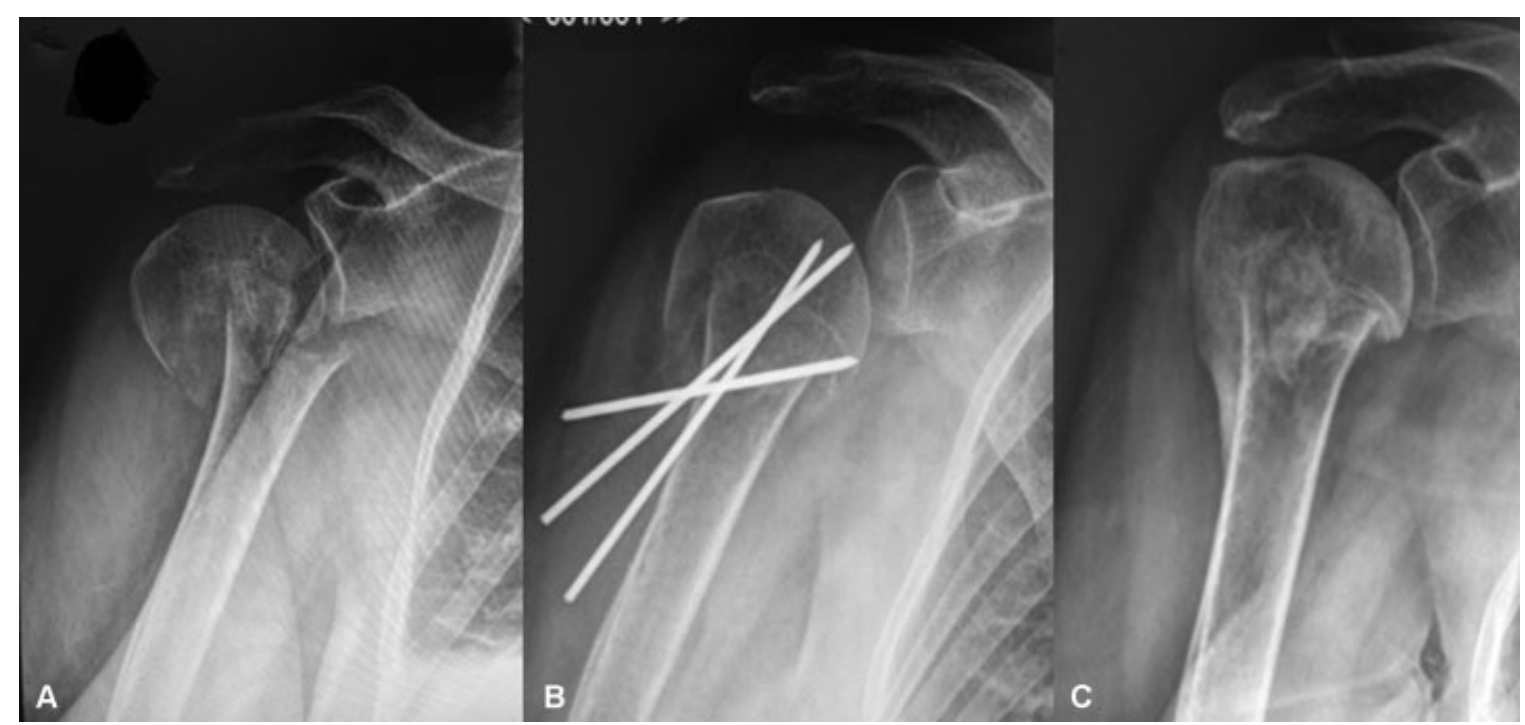

Fig. 5 Radiografia pré-operatória (A); Radiografias pós-operatória com 4 semanas (B); Radiografia pós-operatória com 3 meses (C). 


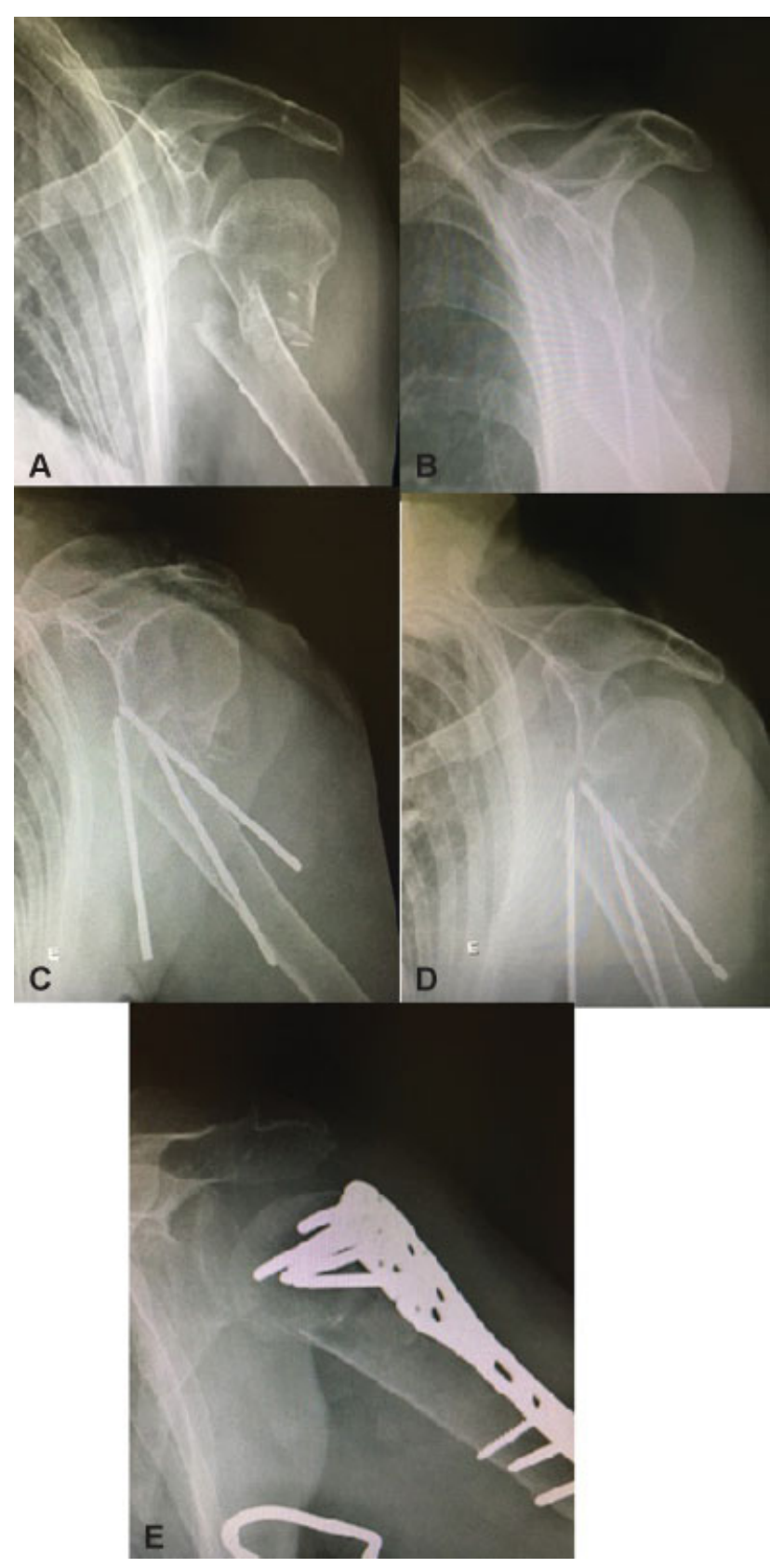

Fig. 6 Radiografia pré-operatória (A, B); Radiografias pós-operatórias com 2 semanas com perda da redução (C, D); Radiografia pós-operatória 2 meses após revisão com placa bloqueada (E).

\section{Discussão}

O tratamento das fraturas do úmero proximal é controverso. Diversas técnicas são descritas que vão desde a imobilização com tipoia até a substituição artroplástica. Diferentes tipos de tratamento em um mesmo padrão de fratura mostram resultados radiográficos e funcionais semelhantes; por isso, nenhum método é considerado padrão-ouro dessa patologia. Na literatura atual, percebemos ainda um considerável índice de complicações relacionadas aos implantes rígidos como perda de redução, não consolidação e cut-out de parafusos que ocorre com certa frequência nas fixações com placa de parafusos bloqueados, seja por mau posicionamento do material, remodelação da fratura ou necrose tardia. ${ }^{10,12,13}$
Tabela 4 Pacientes que perderam a redução

\begin{tabular}{|l|l|l|l|}
\hline \multicolumn{4}{|l|}{ PACIENTES QUE PERDERAM A REDUÇÃO } \\
\hline DESVIO & IDADE & CONSOLIDAÇÃO & CONVÊNIO \\
\hline Medial & 84 & Não Consolidou & PRIVADO \\
\hline Valgo & 62 & Consolidou & PRIVADO \\
\hline $\begin{array}{l}\text { Medial/anterior/ } \\
\text { varo }\end{array}$ & 85 & Consolidou & PRIVADO \\
\hline Medial/Anterior & 74 & Não Consolidou & PÚBLICO \\
\hline Medial/Anterior & 63 & Não Consolidou & PÚBLICO \\
\hline Medial/Varo & 64 & Consolidou & PÚBLICO \\
\hline Varo & 35 & Consolidou & PRIVADO \\
\hline
\end{tabular}

A técnica de fixação percutânea segue os princípios do design de implantes defendidos por Resch, como: a impacção controlada, o pico de direção das forças e o implante semirígido. ${ }^{2}$ Acreditamos que a fixação do colo do úmero com pinos rosqueados permita uma certa acomodação guiada do foco de fratura favorecendo a consolidação. Resch e Hertel descrevem esses dois fenômenos como guide impaction e sintering effect. , $^{2,9}$ Como os pinos são removidos após a consolidação, é permitido colocá-los no intraoperatório numa posição subcondral, local em que o osso da cabeça umeral é mais denso, melhorando sua fixação. A remoção dos implantes evita o risco de complicações tardias como o cutout na epífise proximal. Outra vantagem dos pinos percutâneos, dada sua menor morbidade, é a menor agressão ao tecido muscular e vascular ao redor do ombro, gerando menor dor, menor tempo de internação, melhor cosmese e, principalmente, menos agressão à vascularização do foco de fratura. Como a fáscia clavipeitoral não é aberta, o hematoma da fratura é mantido, o que colabora para a consolidação óssea. Este talvez seja um dos motivos pelos quais percebemos na maioria dos casos a formação de calo ósseo na radiografia de controle entre 3 e 4 semanas, menos comum nas osteossínteses abertas com estabilidade absoluta. ${ }^{14-16}$ Outra vantagem da fixação percutânea com pinos rosqueados é o fato dela ser um método de menor custo do que a fixação com placa e hastes bloqueadas para a fixação das fraturas do colo do úmero.

No nosso serviço, a fixação percutânea com fios rosqueados tem se mostrado como alternativa para o tratamento cirúrgico da fratura do colo cirúrgico na extremidade proximal do úmero, devido aos resultados favoráveis obtidos na avaliação radiográfica dos pacientes, com índice de 90,4\% de consolidação, obtendo taxas de consolidação semelhantes às observadas na literatura. ${ }^{17}$ Observamos que entre os pacientes com $<60$ anos, obtivemos um índice de $100 \%$ de consolidação das fraturas.

Como desvantagem, os pinos rosqueados não proporcionam uma estabilidade tão efetiva à fixação da fratura quanto as placas bloqueadas. Nos casos que perderam a redução, observamos além de um traço mais baixo (em direção à diáfise) uma cominuição do colo tanto da cortical medial quanto lateral, ${ }^{9,10,18}$ indo ao encontro de autores que citam que a redução anatômica da fratura e reformatação do 
Tabela 5 Média em anos dos pacientes que mantiveram e perderam a redução e dos que foram reintervidos

\begin{tabular}{|l|l|l|}
\hline Grupo & $\begin{array}{l}\text { Idade média } \\
\text { em anos }\end{array}$ & $\begin{array}{l}\text { Desvio } \\
\text { Padrão }\end{array}$ \\
\hline Mantiveram a Redução & 56,2 & 18,1 \\
\hline Perderam a Redução & 66,7 & 15,7 \\
\hline Reintervenção & 71,25 & 8,5 \\
\hline
\end{tabular}

suporte cortical medial sejam fundamentais para a consolidação. ${ }^{10}$ Observamos que a média de idade dos pacientes que perderam a redução $(66,71$ anos $\pm 15,7$ desvio padrão [DP]) e dos pacientes que foram reintervidos com outro método $(71,2 \pm 8,5 \mathrm{DP})$ foram maiores que a média de idade do estudo (58,47 anos $\pm 18,3 \mathrm{DP}$ ) (-Tabela 5). Isso corrobora com a literatura de que pacientes mais idosos possuem ossos mais osteoporóticos, tornando-os mais díficil de serem fixados e de manterem redução, seja qual for o implante.

Em comparação a métodos que seguem os mesmos princípios de fixação, como o sistema Humerusblock (implante que agrega um dispositivo na diáfise do úmero que bloqueia os fios), tivemos uma taxa semelhante de reintervenção e revisão com placa bloqueada. ${ }^{19}$ Algumas complicações relativas ao método de fixação podem ser observadas tanto no uso de fios rosqueados, bem como com o sistema Humerusblock, que são a migração proximal ou distal dos fios, sendo necessária sua retirada, se completo o tratamento, ou ao menos o recuo do fio migrado. ${ }^{20}$

\section{Conclusão}

A fixação percutânea das fraturas desviadas do colo cirúrgico do úmero com fios rosqueados pode ser um método de fixação a ser considerado no tratamento desse tipo de fratura, sendo que foram obtidas taxas de consolidação $(90,4 \%)$ e de complicações, ou não consolidação (9,5\%), semelhantes aos observados no uso de outros métodos de fixação. Idade avançada, perda da cortical medial e extensão metafisária foram fatores relacionados ao aumento da perda de redução e possível não consolidação, e devem ser levados em conta ao indicar essa técnica.

Conflito de Interesses

Os autores declaram não haver conflito de interesses.

\section{Referências}

1 Horak J, Nilsson BE. Epidemiology of fracture of the upper end of the humerus. Clin Orthop Relat Res. 1975;112:250-253

2 Resch H. Proximal humeral fractures: current controversies. J Shoulder Elbow Surg 2011;20(05):827-832

3 Tepass A, Blumenstock G, Weise K, Rolauffs B, Bahrs C. Current strategies for the treatment of proximal humeral fractures: an analysis of a survey carried out at 348 hospitals in Germany,
Austria, and Switzerland. J Shoulder Elbow Surg 2013;22(01): e8-e14

4 Resch H, Tauber M, Neviaser RJ, et al. Classification of proximal humeral fractures based on a pathomorphologic analysis. J Shoulder Elbow Surg 2016;25(03):455-462

5 Hertel R. Fractures of the proximal humerus in osteoporotic bone. Osteoporos Int 2005;16(Suppl 2):S65-S72

6 Gupta AK, Harris JD, Erickson BJ, et al. Surgical management of complex proximal humerus fractures-a systematic review of 92 studies including 4500 patients. J Orthop Trauma 2015;29(01): 54-59

7 Resch H, Povacz P, Fröhlich R, Wambacher M. Percutaneous fixation of three- and four-part fractures of the proximal humerus. J Bone Joint Surg Br 1997;79(02):295-300

8 Grubhofer F, Wieser K, Meyer DC, Catanzaro S, Schürholz K, Gerber C. Reverse total shoulder arthroplasty for failed open reduction and internal fixation of fractures of the proximal humerus. J Shoulder Elbow Surg 2017;26(01):92-100

9 Hertel R, Hempfing A, Stiehler M, Leunig M. Predictors of humeral head ischemia after intracapsular fracture of the proximal humerus. J Shoulder Elbow Surg 2004;13(04): 427-433

10 Krappinger D, Bizzotto N, Riedmann S, Kammerlander C, Hengg C, Kralinger FS. Predicting failure after surgical fixation of proximal humerus fractures. Injury 2011;42(11):1283-1288

11 Sukthankar AV, Leonello DT, Hertel RW, Ding GS, Sandow MJ. A comprehensive classification of proximal humeral fractures: HGLS system. J Shoulder Elbow Surg 2013;22(07):e1-e6

12 Ortmaier R, Filzmaier V, Hitzl W, et al. Comparison between minimally invasive, percutaneous osteosynthesis and locking plate osteosynthesis in 3-and 4-part proximal humerus fractures. BMC Musculoskelet Disord 2015;16:297

13 Südkamp N, Bayer J, Hepp P, et al. Open reduction and internal fixation of proximal humeral fractures with use of the locking proximal humerus plate. Results of a prospective, multicenter, observational study. J Bone Joint Surg Am 2009;91(06): 1320-1328

14 Jaura G, Sikdar J, Singh S. Long Term Results of PHILOS Plating and Percutaneous K-Wire Fixation in Proximal Humerus Fractures in The Elderly. Malays Orthop J 2014;8(01):4-7

15 Matziolis D, Kaeaeb M, Zandi SS, Perka C, Greiner S. Surgical treatment of two-part fractures of the proximal humerus: comparison of fixed-angle plate osteosynthesis and Zifko nails. Injury 2010;41(10):1041-1046

16 Seyhan M, Kocaoglu B, Nalbantoglu U, Aydin N, Guven O. Technique of Kirschner wire reduction and fixation of displaced twopart valgus angulated proximal humerus fractures at the surgical neck. J Orthop Trauma 2012;26(06):e46-e50

17 Magovern B, Ramsey ML. Percutaneous fixation of proximal humerus fractures. Orthop Clin North Am 2008;39(04): 405-416

18 Carbone S, Moroder P, Arceri V, Postacchini R, Gumina S. The amount of humeral head impaction of proximal humeral fractures fixed with the Humerusblock device. Int Orthop 2014;38 (07):1451-1459

19 Moroder P, Tauber M, Carbone S, Auffarth A, Resch H. Modern minimally invasive treatment of proximal humeral fractures. Tech Orthop 2013;28:281-286

20 Bogner R, Hübner C, Matis N, Auffarth A, Lederer S, Resch H. Minimally-invasive treatment of three- and four-part fractures of the proximal humerus in elderly patients. J Bone Joint Surg $\mathrm{Br}$ 2008;90(12):1602-1607 